https://helda.helsinki.fi

\title{
Unrealistic assumptions and unnecessary confusions : rereading and rewriting F53 as a realist statement
}

\section{Mäki, Uskali}

Cambridge University Press

2009

Mäki , U 2009 , Unrealistic assumptions and unnecessary confusions : rereading and rewriting F53 as a realist statement . in U Mäki (ed.), The methodology of positive economics : Reflections on the Milton Friedman legacy . Cambridge University Press, Cambridge , pp. 90-116 .

http://hdl.handle.net/10138/232401

acceptedVersion

Downloaded from Helda, University of Helsinki institutional repository.

This is an electronic reprint of the original article.

This reprint may differ from the original in pagination and typographic detail.

Please cite the original version. 


\title{
Unrealistic assumptions and unnecessary confusions: Rereading and rewriting $F 53$ as a realist statement
}

\author{
Uskali Mäki \\ Academy of Finland \\ uskali.maki@helsinki.fi \\ To appear in \\ The Methodology of Positive Economics: \\ Milton Friedman's Essay After Half a Century, edited by Uskali Mäki. \\ Cambridge University Press.
}

\begin{abstract}
It is argued that rather than a well defined F-Twist, Milton Friedman's “Methodology of positive economics" offers an F-Mix: a pool of ambiguous and inconsistent ingredients that can be used for putting together a number of different methodological positions. This concerns issues such as the very concept of being unrealistic, the goal of predictive tests, the as-if formulation of theories, explanatory unification, social construction, and more. Both friends and foes of Friedman's essay have ignored its open-ended unclarities. Their removal may help create new common ground for more focused debate in economics. Here I show how F53 can be reread/rewritten as a socially constructivist fallibilist, and realist statement - in contrast to the received instrumentalist interpretation.
\end{abstract}

Keywords:

Unrealistic assumptions, predictive tests, truth, as-if, unification, underdetermination, social construction

Acknowledgements

This is an expanded and revised version of a paper presented at the conference on F53 at Erasmus University of Rotterdam in December 2003. And that version was a much expanded version of the one presented at the ASSA meetings in Washington DC in January 2003. After the Erasmus conference, it has been presented at the universities of Aix-en-Provence, Freiburg, Athens, and Lund. Useful comments from the various audiences are acknowledged. In his characteristically generous fashion, Milton Friedman offered some written comments that have been consequential. 


\section{Introduction}

Depending on the reader's intellectual preferences and capacities, the experience of reading Milton Friedman's 1953 essay on economic methodology (F53 for short) tends to be either one of relief and conviction or one of irritation and provocation - or perhaps some mixture of these feelings. Many practicing economists have found the message of the essay appealing and liberating, and its arguments convincing. Most specialists in economic methodology, and some practicing economists, have found F53 entirely defective, presenting a dangerously misguided doctrine of scientific theory and method. Both of these responses may be based on an incomplete understanding of the essay, or so I want to argue.

I will argue for two sets of general claims. First, there is no unambiguous doctrine or argument presented in F53. The essay presents no unproblematically identifiable single methodology of economic theorizing. The message of F53 cannot be captured by popular phrases like "Friedman's instrumentalism" or "Friedman's as-if methodology" or "Friedman's argument in support of unrealistic assumptions" or the "F-Twist". This is because F53 also contains strong anti-instrumentalist elements; because "as-if" is itself highly flexible and compatible with a variety of philosophical outlooks; and because F53 argues for unrealistic (and sometimes for realistic) assumptions from sets of mutually incompatible premises. What the reader is served is an F-Mix, a mixture of ingredients many of which are ambiguous and some of which are hard to reconcile with one another. In consequence, a variety of readers with different intellectual tastes will be able to find in F53 their own selection of ideas that they will endorse or oppose.

Second, the flexibility and malleability of the textual material creates room for alternative readings of F53 that challenge its current reified textbook image. In particular, it is possible to develop non-traditional interpretations that might bring many former friends and foes of F53 - or, more precisely, of what has been believed to be the message of F53 - closer to one another, if not unite them entirely. In what follows, I will remove some unnecessary confusions and ambiguities in F53, and by doing so, hope to pave the way for a new sensible reading that will be found much less objectionable by many more readers, practitioners and methodologists alike. To the extent that my rereading fails to be a matter of unbiased discovery of what is already there, hidden in the text of F53, it can 
also be taken as a project of rewriting the essay. It is a matter of rewriting by selection and correction so as to eliminate its flaws and to make it more agreeable to a variety of audiences. On this rereading (or rewriting) F53 emerges as a realist (rather than instrumentalist) manifesto with strong fallibilist and social constructivist sensitivities (in contrast to standard textbook positivism). ${ }^{1}$

One might object by saying that my rereading F53 as a realist and social constructivist statement amounts to a reformulation beyond recognition, or at least to a forced interpretation that does not come naturally. In contrast, my conviction is that what I am about to offer is an obvious interpretation that does come very naturally indeed. My interpretation is more forced when ignoring parts of what F53 seems to be saying, while it is less forced when based just on highlighting elements in F53 that have been overlooked by other readers. But I must grant there is a possible source of bias: the image of economics that emerges in F53 as it is being reread and rewritten here is one that I myself endorse. I am rebuilding F53 as a statement that captures and conveys this rebuilder's own methodological convictions.

A remark is needed on the strategy and motivation of this paper by way of locating it on the map of the various readings of F53 (the map was I outlined in Chapter 2 above). First, mine is primarily an examination of F53 itself rather than explicitly using F53 for some other purpose (though I think it wonderfully serves other useful purposes). Second, my reading goes beyond the narrow logical accounts (in terms of the relationship between 'assumptions' and 'predictions') and incorporates ontological, semantic and social ingredients in the image that F53 conveys of economics. Third, the reading combines normative and descriptive perspectives in that it suggests remedying some flaws of F53 so as to identify and reconstruct its sensible core ideas. Fourth, it attaches extra-economic labels to F53, borrowed from philosophy and science studies. Finally, and importantly, my reading is an exercise in what I called reception methodology: the focus lies on the context of consumption of F53 rather than its production by its author. The context is that of the reader's reception rather than that of the author's intention.

\footnotetext{
${ }^{1}$ This paper follows the line of, and draws on, earlier work such as Mäki 1986, 1989, 1992, 2000, 2003. These papers document my career in the F53 exegesis as one of swimming against the stream.
} 
Reception methodology here amounts to reading F53 from the point of view of its actual and possible interpretations and influences among the relevant audiences.

It is part of my receptionist reading of the essay that I make no claims about the author's intentions: I am not after Friedman's authentic convictions or anything related to "what Friedman really meant" (in fact I am not convinced there are such things there to be discovered, at least in F53 alone). ${ }^{2}$ The focus here is on the text of F53 while the author's intentions and beliefs are bracketed. As a technical expression of this I am not referring to the author but rather to the text: I am not saying "here Friedman argues..." but rather, "F53 claims..."; not "Friedman's opinion seems to be..." but rather, "F53 can be read as..." and so on. My attempt to "rewrite" F53 so as to make it more acceptable to various audiences is also independent of anything that Milton Friedman the author might have believed or intended, or of whether he would accept the rewritten version of F53 as sound (even though I believe he would have ample reason to accept it).

Let it be added that the receptionist reading of F53 is also consistent with, and supported by, Milton Friedman's personal strategy in relation to F53, as repeated in his statement at the end of this volume: he has chosen not to respond to any commentaries on F53 by others, but has rather let the text live its own life in the intellectual realm, at the mercy of its readers, as it were.

\section{Assumptions and predictions, and the non-predictivist torso}

If there is one basic thesis conveyed by F53, it is this: economic theories should not be judged by their assumptions but by their predictive implications - and in particular, the unrealisticness of the assumptions of a theory is no reason for complaint or worry about the theory. The methodological advice given to economists by F53 appears simple: when assessing a theory, don't pay separate attention to its assumptions, instead focus on the predictions it yields. As the much-cited statement puts it, "the only relevant test of the validity of a hypothesis is comparison of its predictions with experience. The

\footnotetext{
${ }^{2}$ The only way to determine what Friedman's possible authentic methodological convictions are is to read F53 together with his other methodological remarks conjoined with a scrutiny of the implicit methodology of his work in economics. This is a very legitimate historical and analytical project, but it does not lie at the heart of my reception methodology.
} 
hypothesis is rejected if its predictions are contradicted ('frequently' or more often than predictions from an alternative hypothesis); it is accepted if its predictions are not contradicted" (F53, 8-9). This passage makes three important points about testing scientific theories: testing is by predictive implications, not by assumptions; failed predictions play a key role: acceptances are just failures to be rejected; and testing is comparative: what matters is the predictive performance of a theory relative to that of alternative theories. ${ }^{3}$

It is clear that the main focus of attention of the text of F53 is on assumptions, not on predictions. The historical context of F53 essentially contained various attacks against some of the key assumptions of marginalist theory, blaming them for harmful unrealisticness. F53 sets out to convince the skeptical or hesitant reader that unrealistic assumptions are just fine, and the arguments of F53 revolve around this idea. Yet, in some cases the reader would expect to be shown the full force of the basic thesis in its entirety, including the role of comparative predictive performance.

Consider the treatment of Edward Chamberlin's theory of monopolistic competition. F53 does not hide its hostility towards Chamberlin's theory - an inferior theory that economics does not need at all in addition to the superior simple models of perfect competition and perfect monopoly. The argument of F53 is straightforward: the creation of the theory of monopolistic competition was "explicitly motivated, and its wide acceptance and approval largely explained, by the belief that the assumptions of 'perfect competition' or 'perfect monopoly' said to underlie neoclassical economic theory are a false image of reality" (15), and it is this misguided motivation and flawed basis of acceptance that speaks against the theory. What is noteworthy is that there is no appeal here to the superior predictive capacity of Friedman's favorite theories in contrast to the predictive failures of Chamberlin's theory. The realisticness or unrealisticness of assumptions was not supposed to matter, but it seems they do, after all. The key thesis of F53 is thereby turned into a modified non-predictivist torso: hail unrealistic assumptions, proscribe against the pursuit of realistic assumptions.

\footnotetext{
${ }^{3}$ In these formulations, one may choose to hear echoes of Popper's doctrine of refutation based on failed predictions as well as Lakatos's doctrine of comparative and dynamic predictive performance. The English translation of Popper's Logik der Forschung appeared in 1959 (Popper 1959), while Lakatos's relevant essays were published a decade or so later (e.g. Lakatos 1970).
} 
This I believe is how many practicing economists have received the message of F53. This explains its emancipatory effect on economists: it helps liberate those employing models with unrealistic assumptions from a sense of unease (see Mayer 1993). This also suggests why there is an easy link between F53 and the defense of "blackboard economics" that is mathematically highly refined and rigorous but accused for being unconnected to real world facts and issues (cf Hands 2003 and in this volume). Whatever the methodological preferences of the author of F53 - in fact we know Friedman is an opponent of formalistic blackboard economics - it is not surprising to see appeals to F53 in justification of the assumptions that help create the model worlds on the blackboard. From the point of view of the context of consumption, it is the reception and use of F53 that matters. A tension between the author's intended methodology and the use of the torso version of the basic thesis of F53 does not lie within the purview of reception methodology.

\section{Unrealisticness as irrelevant and as a virtue}

Friedman's claim about unrealistic assumptions appears at least in two versions: one is in terms of irrelevance, the other is in terms of virtue. The weaker version is the claim that unrealisticness is irrelevant for the goodness of theory: that it does not matter even if a theory's assumptions are unrealistic. Now it is important to see that a consistent irrelevance thesis would have to imply that the actual degree of both unrealisticness and realisticness does not matter. In other words, no matter how unrealistic or realistic the assumptions of a theory, this is irrelevant to the assessment of the theory, since those properties are not among the ones we should measure when evaluating a theory. But the bulk of F53 is not symmetric in this way, thus not in line with the weak version: the essay keeps stressing that it is high degrees of unrealisticness that do not matter. As the attack in F53 against more realistic behavioral assumptions and the theory of monopolistic competition indicates, the pursuit of high degrees of realisticness is

Friedman had learnt about Popper's falsificationist views when personally meeting him in the late 1940s. 
regarded as a serious demerit of both a theorist who does so and of a theory that is pursued. This contradicts the weak thesis in terms of irrelevance.

The strong version acknowledges the asymmetry of F53 and does it in a radicalized manner: it is the claim that unrealisticness is a virtue, that the better the theory, the more unrealistic it is in its assumptions. Many readers have found the strong version unacceptable, even outrageous. Indeed, as a general rule, it must be mistaken. Even in some cases where violating the truth may seem recommendable - such as in assuming a vacuum for falling cannon balls and assuming profit maximization for certain purposes - the strong version goes too far. F53's examples of excellent scientific theories assume zero air pressure and profit maximization. The strong version suggests that there might be even better theories that assume that air pressure is infinitely large and that businessmen aim at maximizing their losses - these assumptions would be more unrealistic than the ordinary ones. But obviously, such unrealistic assumptions would not be epistemically virtuous, thus the strong version is questioned.

There are obvious cases in which both versions appear questionable. In the study of the used cars market, it is neither irrelevant nor virtuous for a theory to falsely assume that information is symmetric, and in the study of the computer software industry, one is not well advised to assume diminishing returns. Qualifications and further conditions are needed: neither version of Friedman's thesis can be defended as a general principle. I will have more to say about this in later sections.

\section{Indirectly testing approximation to the truth}

On closer scrutiny, it appears that F53 does not subscribe to either of the above versions of the thesis consistently or without qualifications. Indeed, the truth of assumptions appears as a relevant issue after all. F53 indicates an interest in truth when suggesting that truth is something to be estimated: indeed, predictive tests serve as indirect tests of the approximate truth of assumptions. The required degree of approximation is not simply to be maximized (or minimized!) but is relative to the purposes that the theory is supposed to serve: "the relevant question to ask about the 'assumptions' of a theory is ... whether they are sufficiently good approximations for the purpose at hand" (15). So there is a relevant question to ask about assumptions, and the question is about their approximate 
truth. The way to measure whether the required degree of approximation has been achieved is to put the theory in predictive test:

Complete 'realism' [realisticness] is clearly unattainable, and the question whether a theory is realistic 'enough' can be settled only by seeing whether it yields predictions that are good enough for the purpose in hand or that are better than predictions from alternative theories. (41)

This implies that the unrealisticness of assumptions is not an irrelevant issue at all, something to be ignored. On the contrary, one is advised to pay attention to their actual degree of realisticness and to judge whether it is sufficiently high for the purposes at hand. It appears that two kinds of considerations shape these judgements: pragmatic and ontological. Pragmatic considerations enter in the form of purposes: the appropriate degree of (un)realisticness is relative to the purposes for which the theory is put.

Ontological considerations are concerned with the causal powerfulness of various factors: some factors are too weak to be included in the model, while others play major causal roles and should be included. In other words, it all depends on the difference those factors make:

Why is it more 'unrealistic' in analyzing business behavior to neglect the magnitude of businessmen's costs than the color of their eyes? The obvious answer is because the first makes more difference to business behavior than the second; but there is no way of knowing that this is so simply by observing that businessmen do have costs of different magnitude and eyes of different color. Clearly it can only be known by comparing the effect on the discrepancy between actual and predicted behavior of taking the one factor or the other into account. (33)

Indeed, not only is it "more unrealistic" to ignore the magnitude of businessmen's costs than the color of their eyes, but such unrealisticness also matters a great deal. It is advisable to consider costs - but not the eye color - because they make a difference for the phenomena being explained. And to determine whether or not they make a difference relative to some purpose requires checking the predictive implications. Another way of putting these ideas is to say that some of the assumptions of a theory are to be paraphrased as statements about the negligibility of a factor, and that predictive tests are a 
way of assessing such claims about negligibility (Musgrave 1981; Mäki 2000). I will return to this idea in a later section.

\section{Testing by assumptions and testing by predictions}

F53 itself, and indeed most of the commentators on the issue, draw a distinction between testing a theory by its assumptions and testing it by its predictions. Accordingly, the participants in the debate are classified as the "assumptionists" and the "predictivists", the latter often also called instrumentalists (I'll come back to the uses and abuses of this label later). The assumptionists are supposed to test the assumptions "directly" and thereby to test the theory while the predictivists test the theory "indirectly" via its predictive implications.

Without qualifications, this is a misleading distinction. No "direct" testing of assumptions is available. This should be easy to see: when one seeks to test an assumption, one has to construe an argument in which that assumption serves as one of the major premises and which entails a predictive implication that one then compares with evidence. When testing the assumption of transitive preferences, one conjoins it with several other assumptions and draws implications concerning choice behavior, and then checks those implications against, say, experimental data.

I think the intended difference is between two or more domains of testing by implications. Economists hold different views as to the relevant domain of data that should be consulted as evidentially relevant. Thus, some hold the view that the maximization assumption is to be tested against the data concerning the individual behavior of business managers. Others believe the assumption and the theory of which it is a constitutive element, should only be tested by drawing implications concerning market data. In both cases, one tests by predictive implications.

\section{The whole truth and nothing but the truth}

F53 uses a variety of expressions to refer to species of unrealisticness. These include "descriptively false", "descriptively unrealistic', and "descriptively inaccurate" (pp. 1415). None of them is being defined in F53 other than through examples. But the 
examples reveal fatal ambiguities. In particular, F53 conflates falsehood proper (violation of "nothing-but-the-truth") and incompleteness or narrowness (violation of "the whole truth"). These are separate notions, but F53 fails to keep them separate - and so does much of other commentary on the realisticness issue. In particular, the violation of the whole truth does not imply the violation of nothing but the truth about some part of the whole. Here we come across with one major limitation of the logical accounts: they miss the distinction between the whole truth and nothing but the truth, focusing just on the latter and dealing with the former as if it were reducible to the latter.

Consider an example. One of the targets that F53 seeks to rebut consists of the criticisms of the maximization assumption in the "marginalist controversies" launched by Hall and Hitch in the UK and Lester in the US. Friedman invites the reader to consider "the recent criticisms of the maximization-of-returns hypothesis on the grounds that businessmen do not and indeed cannot behave as the theory 'assumes' they do" (31). F53 here formulates the message of the criticism as the charge that the maximization assumption violates nothing but the truth about business behavior: businessmen do not, perhaps cannot, behave the way they are assumed to behave.

Right after this, F53 sketches "a completely 'realistic' theory of the wheat market" that would have to mention the color of the traders' and farmers' eyes and hair, antecedents and education, the physical and chemical characteristics of the soil on which the wheat was grown, the weather prevailing during the growing season, etc. etc. (32). Another notion of realisticness is implied by such a "completely realistic theory": deviations from such a "theory" by leaving out various details would be a matter of violating "the whole truth" about a subject matter. One can easily imagine any number of further details that would have to be mentioned in such a "theory" of the wheat market, including the detailed career of the causal and constitutive ancestors of each farmer, trader, parcel of land, and seed of wheat during the process of cosmological, geological, biological, and cultural evolution billions of years backwards.

F53 implies a close connection or sameness between these two notions of truth when suggesting that to criticize the maximization assumption is as ridiculous as to insist on building such a completely realistic theory. The reasoning is not sound: it is trivial that nobody will insist on having such a "theory" - but it also should be trivial that this has nothing to do with the insistence that it is no recommendation for the maximization of 
returns assumption as such that it violates nothing but the truth. The violation of the whole truth (which is unavoidable) implies nothing about the violation of nothing but the truth (which is avoidable). ${ }^{4}$ Only a small fraction of all facts (even of the causally efficacious ones) are mentioned by any theory, hence the violation of the whole truth; but statements about those facts - such as whether actors in the wheat market are maximizers - are not necessarily false. One cannot justify false assumptions by citing the trivial fact that all theories are necessarily incomplete.

F53 thus conflates the whole truth with nothing but the truth. This conflation may be seen to serve a rhetorical function: just like every sensible person, my dear reader, you surely agree that the insistence on a "completely realistic theory" is undesirable, therefore you are naturally reasonable enough also to agree that to criticize the maximization assumption for falsehood is misguided.

Why is it important to resist this conflation? Keeping the two notions distinct has implications for the assessment of the basic thesis of F53. One may argue that good theories violate the whole (or comprehensive) truth in that they isolate just narrow slices of the world and leave out most of it - such as most of the things listed in the "completely realistic theory" of the wheat market. One may go further and argue that, subject to further (perhaps ontological and pragmatic) constraints, the more a theory leaves out the better it is. One may also point out that such theoretical isolations are often accomplished by way of false idealizing assumptions (such as those of a vacuum or homogeneous goods) that help exclude factors (such as actual air pressure or product differentiation) that are viewed as irrelevant for a problem at hand. These are some of the ways in which good theories may be unrealistic. More about this in a moment.

\section{Assumptions and their roles: vacuum and maximization}

Here is another limitation of the logical accounts: they miss a relevant and sufficiently rich idea of sentence role. In the logical accounts, the only conceivable roles of sentences are those of premises and conclusions in logical arguments. In order to understand economic theorizing, we need to have a more qualified notion of role.

\footnotetext{
${ }^{4}$ Many qualifications would have to be added to the two claims about avoidability.
} 
One of F53's famous examples is Galileo's law of freely falling bodies. In the formulation used, the law states that $\mathrm{s}=1 / 2 \mathrm{gt}^{2}$. One of the assumptions behind the law is that of a vacuum:

[1] $\quad$ Air pressure $=0$

F53 argues that for many purposes (such as predicting the fall of a cannon ball) it does not matter even if the vacuum assumption is false. It then uses this as an analogy in its defense of the maximization assumption:

[2] Producers and traders pursue maximum expected returns.

F53 argues that just as, in many cases, it does not matter if the vacuum assumption is false, it does not matter even if the maximization assumption is false. This argument exploits a mistaken analogy, based on ignoring the roles that these two assumptions [1] and [2] are supposed to play within their home theories.

The purpose that assumption [1] serves is similar to that of [3]:

[3] All forces other than gravitation $=0$

Assumptions [1] and [3] are idealizations. They idealize by assuming that certain forces or causes are powerless, they have zero strength: no air pressure, no magnetic forces, no winds, no other kinds of pushes and pulls such as the pull of the Moon. The assumptions are "unrealistic" in that, in the presently actual world, [3] is always false (for example, the pull of the Moon, the Sun, and other planets are always there), while [1] is mostly false but may sometimes be true if made true (vacuum conditions can be artificially created). By means of such falsehoods, a model is constructed in which all causes of the behavior of a falling body except for one are neutralized. The one cause that remains nonneutralized is the gravity of the Earth. The idealizing assumptions play the important role of helping to isolate the impact of gravitation from other causal influences. 
What about the profit maximization assumption? Is it also an idealization that helps isolate one cause while neutralizing the influence of other causes? One manifest difference between [2] and [1]\&[3] is this: while [1]\&[3] explicitly refer to the factors that they serve to eliminate or neutralize, [2] does not seek to neutralize what it is about, namely profit maximization. Indeed, rather than removing the impact of profit maximization, it establishes profit maximization as a major motive of economically relevant behavior. Instead of saying agents do not maximize, it says they do. The analogy between maximization and vacuum seems to collapse.

If one were to formulate an analogue of assumptions [1] and [3] in Galileo's law, it would not be [2] as F53 suggests, but rather something like

[4] All other motives except the maximization motive have zero strength.

This is an idealization that helps isolate the motive of the maximization of expected returns from all other possible motives (such as moral deliberations, sense of security, fairness, reputation, maximization of sales and/or market share, etc.) in order to make it possible to examine the impact of profit maximization on market phenomena in the absence of such other motives. Maximization would be isolated as the motive rather than just $a$ motive among others. Now one may argue that this indeed is intended already by assumption [2]: by assuming that firms are motivated by expected maximum returns one thereby assumes that they are motivated by nothing but expected maximum returns. In other words, assumption [2] is intended to have assumption [4] as its implicit component. But this does not save the suggested analogy from my rebuttal. In our more literal moods, we might want to say that the profit maximization assumption is a combination of [2] and [4]. This will help us retain the claim that there is an analogy between [1] and [4] but not between [1] and [2].

The analogue of component [2] of the maximization assumption in Galileo's law is not the vacuum assumption but instead something like 
[5] The gravitational field of the Earth pulls physical objects by force $\mathrm{g}$.

It is the gravitation of the Earth that is being isolated by means of idealizing assumptions [1] and [3]. Likewise, it is the drive to maximize that is being isolated by means of idealization [4]. Insofar as truth and falsehood are concerned, it is an entirely different matter for "assumptions" [2] and [5] to be true or false, and for assumptions [4] and [1]\&[3] to be true or false. Moreover, the truth or falsehood of assumptions [1] and [3] does not imply anything about the truth of [5]. Statement [5] may be true even though [1] and [3] are false, and even if they are false to the extent that Galileo's law were to yield radically incorrect predictions, such as in the case of a falling feather in the weekend market of my hometown plaza.

If the analogy between [2] and [5] were to be adequate in relevant respects, one might argue that nothing follows from the acceptability, believability, or truth of [4] for the respective attributes of [2] - in analogy with the similar connection between [1]\&[3] and [5]. One might then say that agents may be profit maximizers even though this is not the only thing that motivates them in business transactions: [2] might be true even if [4] is false. Obviously, this is not at all evident, and certainly would invite a lot of further qualifications. ${ }^{5}$ Anyway, the point relates to the important distinction to be made next.

\section{Truth of assumptions and truth of theory}

Most commentaries of F53 are based on the presumption that one can determine the truth-value of a theory or model by way of checking the truth-values of its assumptions. This is because the truth-value of a theory is a function of the truth-value its assumptions, such as the truth-value of a theory being equal to the truth-value of the conjunction of its assumptions. On this view, the falsehood of assumptions can be taken to imply the falsehood of the theory. F53 admits that many of the central assumptions of the economic

\footnotetext{
${ }^{5}$ The argument in the text about profit maximization and its analogues is in terms of motives. Qualifications would be needed if other versions of the profit maximization assumption were employed, such as one in terms of deliberate marginal calculations and their implementations in maximizing behavior; and another in terms of behavior that ends up with maximizing the profits of a firm regardless of the motives and mental operations of its managers.
} 
theories challenged by their critics are false. This has been interpreted by later commentators as implying a commitment to a special kind of instrumentalist view of theory: economic theories and models are false instruments of prediction (e.g. Wong 1973, Boland 1979, Caldwell 1992).

F53 itself performs better, and we can see this if we look a bit more carefully at the relevant formulations. F53 uses expressions such as a theory being "descriptively false in its assumptions" (14). We are not compelled to read this as admitting that theories with false assumptions are false. Indeed, it has been my contention that theories with false assumptions may be true, and that realism (as a theory of theories) is perfectly comfortable with unrealistic assumptions (e.g. Mäki 1992). The truth value of a theory cannot be read off the truth values of its assumptions. The key to understanding the gap between the two is to ask the question: what is the theory about, what claim does it make about the world, if any? A theory may be true about the functioning of some important causal factor while making false assumptions about the existence and functioning of other factors. Galileo's law is true about, well, what it is about: namely the causal role of gravity in shaping the behavior of falling bodies. The core assertions of the law are not about air pressure, magnetic forces, or the shape of the Earth, but about the gravitational field of the Earth in relation to freely falling bodies. False assumptions about factors other than gravity imply nothing about the truth-value of the law statement itself.

Falsehood is a friend of truth. Not only does the concept of truth require that of falsehood: what is not true is false. Not only does truth often emerge as false beliefs are rejected and replaced by true ones. My somewhat radical statement is that one may claim to have identified significant truths about the world by using a theory with false assumptions. In such cases one characteristically uses those idealizing assumptions to eliminate from consideration some less significant factors and thereby isolates, and helps focus on, what are believed to be the most significant causes and relationships. This is an important feature of the methodological image of theory and method held by many practicing economists. This is part of the realism I am reading in, or writing into, F53. 


\section{Manipulation of truth-values by paraphrasing assumptions}

The previous section suggested that a model with false assumptions may be true. Let us next develop another way of reconciling truth and falsehood by reconsidering the falsehood of assumptions from a different perspective. This is based on Alan Musgrave's contribution (Musgrave 1981) and my suggested modifications and elaborations (Mäki 2000). The trick is to relocate truth claims by paraphrasing assumptions. The point is this: one should not criticize what appears as a false assumption without understanding what assertion is intended when using it. The previous section focused on the truth claims made by means of models, while here the focus lies on the truth claims intended in making assumptions.

On a correct understanding of what claims are intended when putting forth this or that assumption, assumptions taken as such claims should be true. If needed, one uses a paraphrase to determine the intended claim when making an assumption. When suitably paraphrased, the assumptions of a theory had better be true. Such paraphrasings include turning an assumption into an assertion of the negligibility of a factor or of the applicability of a model to a domain of phenomena. The vacuum assumption in connection to predicting the behavior of a freely falling cannon ball can be paraphrased as the statement that the impact of actual air pressure is negligible, given one's purposes (while it is not negligible in the case of a falling feather): the deviation from the truth is negligibly small, or the degree of approximation to the truth is sufficiently close. Such statements about negligibility ("the causal significance of deviations from the vacuum is negligible for the purpose at hand") should be required to be perfectly true - while the non-paraphrased assumptions ("there is a vacuum") provide more (even much more) or less distant approximations to the truth. This difference is implicit in F53.

F53 says that for certain purposes (such as prediction with a certain accuracy) and for certain falling bodies (such as cannon balls), the actual impact of air resistance is negligibly small, therefore assuming that those bodies fall in a vacuum is all right and Galileo's law applies. F53 also suggests that for some bodies, such as feathers, air resistance makes a non-negligible difference, therefore the vacuum assumption is not justified. This suggests using the vacuum assumption as an applicability assumption 
saying that the simplest formulation of the law of freely falling bodies only applies in cases where actual air pressure has negligibly small consequences for the behavior of falling bodies. An applicability assumption makes claims about the applicability of a model or law statement to some domain. Claims about applicability had better be true, just as claims about negligibility should be true.

These are all very reasonable claims and they indicate an appreciation for the role that judgements about matters of fact play in theorizing. These considerations do not fit smoothly with an instrumentalist view of theory. They are rather realist considerations, but they do not require the non-paraphrased (yet paraphrasable) assumptions to be perfectly or even moderately realistic. Yet they stress the principle that the appropriately paraphrased assumptions had better be realistic.

In this connection, it is noteworthy that the specific "Marshallian" (or antiWalrasian) methodological preferences that lie behind F53 have little appreciation for considerations of formal tractability as a constraint on theorizing - in contrast to more mathematically oriented styles of doing economics (on tractability issues, see Hindriks 2005). The latter might include paraphrasing an assumption that, taken as such, is false, as an assertion that says: the use of the assumption enhances the formal tractability of the model. Such an assertion is about the intrinsic properties of a model in relation to the available formal tools and skills, not about the properties of the real world. Even if this strategy were to generate a perfectly true assertion, it would not satisfy those "Marshallian" methodological preferences. This is also in line with a realist outlook: true tractability assumptions do not describe facts about economic reality, nor are they easily justifiable by reference to such facts.

The above reasoning was not tied to any particular assumptions. But there is one particular assumption that F53 briefly discusses and suggests justifying in a way that involves the paraphrase technique. Again, the idea is that the assumption only appears to make false assertions about the world, but when suitably paraphrased, falsehood is replaced by truth. The assumption in question is profit maximization, understood literally as dealing with goals and computations taking place in businessmen's extended minds: by going through a series of marginalist calculations, they consciously infer what the maximizing behaviour is, then take action accordingly. The paraphrase of this assumption dispenses with the decision procedures behind overt behaviour, and makes claims about 
that behaviour only. The assertion now made is about behaviour that ends up with maximum profits - rather than about profit maximizing motives and calculations. F53 indicates optimism that so paraphrased, the assumption has a fair chance of being (at least approximately) true.

Moreover, F53 suggests justifying the assumption in terms of an evolutionary argument that offers "indirect evidence" in its support. The mechanism of natural selection in competitive markets ensures that only firms exhibiting profit maximizing behaviour survive: “... unless the behavior of businessmen in some way or other approximated behavior consistent with the maximization of returns, it seems unlikely they would remain in business for long" (22). On this reasoning, it does not matter what precise computational processes have preceded and generated overt business behaviour. Businessmen only behave as if they were deliberately maximizing. The realist response to this argument would be in two parts. First, just as Koopmans (1957) insisted long ago, the evolutionary argument should be taken to imply the obligation to develop an account of the evolutionary mechanisms themselves as one core target of theory. Second, one should have an account of the structure of the 'as-if' locution that connects profit maximization as a deliberately pursued goal and as manifest behaviour.

\section{Two kinds of as-if}

It seems to be generally believed that the use of the 'as-if' locution in formulating theoretical claims commits one to an anti-realist (fictionalist or instrumentalist) view about scientific theory. This is a mistake. The as-if formulation is a flexible tool that can be used for expressing a number of ideas about a theory and its relationship to the world. F53 itself appears to be of two minds as to the import of 'as-if'. It is one thing to say that

[a] phenomena behave as if certain ideal conditions were met, namely conditions under which only the theoretically isolated real forces are active;

and it is quite another thing to say that 
[b] phenomena behave as if those forces were real.

The difference between the two is striking and has important philosophical implications. Of the two, [a] is in the spirit of realism, while [b] invites a fictionalist and instrumentalist reading. Formulation [a] says that the behavior of certain phenomena is shaped by a real force isolated by the theory, and that it is to be treated without considering the role of other factors, suggesting that those phenomena behave as if the theoretical isolation were materialized also in the real world. This is what F53 is effectively saying in one of its general passages:

A meaningful scientific hypothesis or theory typically asserts that certain forces are, and other forces are not, important in understanding a particular class of phenomena. It is frequently convenient to present such a hypothesis by stating that the phenomena it is desired to predict behave in the world of observation as if they occurred in a hypothetical and highly simplified world containing only the forces that the hypothesis asserts to be important. (40)

That this is in line with [a] should be evident. There are a number of causally relevant forces in the world. But they are not equally relevant, thus the economist proceeds by assuming that it is just the most powerful forces that shape the explanandum phenomenon, that they function in isolation from all other forces: the phenomenon occurs as if nothing but those powerful forces were in operation. The reality of none of these forces is denied. If one wants to identify a fiction here, it is the isolation that is a fiction, not the isolated forces (Mäki 1992, 2004).

In its concrete illustrations, on the other hand, F53 is closer to formulation [b]. The imaginative hypothesis of the leaves of a tree is the most striking of its examples. F53 suggests the "hypothesis that the leaves [around a tree] are positioned as if each leaf deliberately sought to maximize the amount of sunlight it receives, given the position of its neighbors, as if it knew the physical laws determining the amount of sunlight that would be received in various positions and could move rapidly or instantaneously from any one position to any other desired and unoccupied position." (19) However, "so far as we know, leaves do not 'deliberate' or consciously 'seek', have not been at school and learned the relevant laws of science or the mathematics required to calculate the 
'optimum' position, and cannot move from position to position" (20). In contrast to the general statement cited above, this suggests that the forces cited after 'as-if' are not real: we are fully aware of the fictionality of the forces postulated, and this awareness is expressed in terms of the 'as-if'. This represents a fictionalist use of 'as-if'. Our realist rereading of F53 will ignore this passage.

Note that the fictionalist use of the 'as-if' rules out yet another way of using the 'as-if', namely its epistemological use in expressing uncertainty. In this case, what follows an 'as-if' is a hypothesis describing one possible causal trajectory, and it is taken to be the task of further research to establish whether what seems possible is also actually the case. This is not an option for the fictionalist use of the 'as-if' but can (and should) be accommodated in a realist account. (Mäki 1998)

\section{Theoretical isolation and explanatory unification}

In line with the realist use of the 'as-if', F53 contains passages suggesting that theory construction is a matter of theoretical isolation whereby economists "abstract essential features of complex reality" (7). This is a widely endorsed idea in economics and elsewhere: the real world is complex, therefore we need to build simple models that theoretically isolate causally significant aspects of the world. This general idea also provides justification for the employment of false assumptions in such models. The simplest version of Galileo's law isolates the significant impact of gravity on the falling body. It excludes the impact of other forces by false idealizations such as those of vacuum, absence of magnetic forces, flatness of the earth. On this picture, it is the task of false assumptions to help isolate major causal factors.

F53's remarks about these matters seem to be ontologically motivated. The economist is supposed to theoretically isolate "essential features of complex reality" which suggests that it is a (higher order) feature of reality itself that some of its features are essential. The same idea is conveyed by another important passage in F53. This one introduces the realist notions of deceptive appearances and ontologically grounded explanatory unification:

A fundamental hypothesis of science is that appearances are deceptive and that there is a way of looking at or interpreting or organizing the evidence 
that will reveal superficially disconnected and diverse phenomena to be manifestations of a more fundamental and relatively simple structure. (33)

One is invited to read this roughly as follows. The world is, really, not as it appears to be: appearances are deceptive manifestations of more fundamental structures. Scientific theories are required to capture those fundamental structures. This suggests a distinction between reality and appearance. To this the passage adds the notion of explanatory unification, the idea of theoretically describing what appears diverse as really constituting a unity. A theory unifies apparently disconnected phenomena by showing them to be manifestations of the same fundamental structure. Unification amounts to showing that those phenomena are really connected and only apparently disconnected, and this is accomplished by successfully representing how things are related in the way the world works. This is a matter of ontological unification (Mäki 2001), and is only attainable by using a theory that truthfully manages to isolate the key causes and relations underlying such apparently disparate phenomena. This gives us a realist notion of explanatory unification.

Once again, the leaves-of-a-tree example offers a different story. It, too, appeals to the virtue of unification. Indeed, in F53 the hypothesis that the leaves of a tree actively and deliberately seek some goals emerges, after all, as inferior precisely because it does not exhibit the virtue of unifying power. The alternative hypothesis - according to which the leaves only passively adapt to external circumstances - is presented as superior because it unifies, because "it is part of a more general theory that applies to a wider variety of phenomena, of which the position of leaves around a tree is a special case, has more implications capable of being contradicted, and has failed to be contradicted under a wider variety of circumstances" (20). This passage, taken alone, would be compatible with the realist notion of ontological unification. But the account given by F53 about this example departs from the realist view after all: even though F53 is explicit that the power to unify constitutes a significant criterion of theory choice, realist hopes are undermined as no role is supposed to be played by what is justifiably believed to be the case in the real-world causation of leaves' behaviour. The argument here in F53 ignores the fact that the core assumptions of the "activist" hypothesis are false and those of the "passivist" hypothesis are more realistic in isolating the key lines of that causation (20). What is 
claimed to be decisive is not related to what causal processes are actually believed to take place in nature, but rather to the range of conclusions that can be derived from the two hypotheses and the range of theoretic connections they exhibit. What the argument appeals to is derivational unification (Mäki 2001): the power of theories to generate large sets of logical conclusions. The power of theories to describe what is really going on and what real unity there might be in the world - is viewed as irrelevant, thus this argument is a non-realist one. ${ }^{6}$ In our realist rereading of F53 we simply put its treatment of this example aside.

\section{Underdetermination and theoretical virtues}

One of the troubling logical features of theorizing is that theory choice is underdetermined by empirical evidence. No body of evidence is sufficient for determining a unique choice of theory. Yet another reason for reading F53 as an up-todate statement of methodology is that it indicates admirable awareness of the underdetermination issue: ${ }^{7}$ predictive success of a theory "is not by itself a sufficient criterion for choosing among alternative hypotheses" (9). F53 then explains why this is so:

Observed facts are necessarily finite in number; possible hypotheses infinite. If there is one hypothesis that is consistent with the available evidence, there are always an infinite number that are. For example, suppose a specific excise tax on a particular commodity produces a rise in price equal to the amount of the tax. This is consistent with competitive conditions, a stable demand curve, and a horizontal and stable supply curve. But it is also consistent with competitive conditions and a positively or negatively sloping supply curve with the required compensating shift in the demand curve or the supply curve; with monopolistic conditions, constant marginal costs, and stable demand curve, of the particular shape required to produce this result; and so on indefinitely. (9)

\footnotetext{
${ }^{6}$ In the philosophy of science, the current interest in explanatory unification started evolving after Michael Friedman's 1974 article and Philip Kitcher's articles in 1976 and 1981.

${ }^{7}$ The two classical statements of the underdetermination issue were published at about the same time as F53. Quine's "Two dogmas of empiricism" had appeared in 1951, while the English translation of Duhem's book was to be published in 1954 (the French original appeared in 1904).
} 
This is to say that the observational implications of an infinite number of alternative hypotheses are identical; in the example, they all imply a rise in price equal to the tax. F53 thus subscribes to what is generally recognized as the thesis of observational equivalence between theoretical hypotheses. This thesis has opened up a strategic opportunity for anti-realists to undermine realist hopes for rationally believing scientific theories as true. Such anti-realists have used the opportunity by inferring from the thesis of observational equivalence to the thesis of epistemic equivalence: all the observationally equivalent theoretical hypotheses are also equally believable. Given any number of observationally equivalent hypotheses, there is no rational way of believing one of them to be true or closer to the truth than the others (or even the stronger thesis that none of them actually is true or closer to the truth). The choice between epistemically equivalent hypotheses is epistemically arbitrary and must be based on non-epistemic factors. Theory choice is not only empirically undecidable but also epistemically undecidable.

F53 goes some way towards conceding epistemic equivalence and arbitrariness as an implication of observational equivalence: "The choice among alternative hypotheses equally consistent with the available evidence must to some extent be arbitrary..." (10). Immediately after this F53 invokes extra-empirical virtues as criteria of making nonarbitrary (or at least less arbitrary) choices between observationally equivalent theories: "...there is general agreement that relevant considerations are suggested by the criteria 'simplicity' and 'fruitfulness', themselves notions that defy completely objective specification. ... Logical completeness and consistency are relevant but play a subsidiary role..." (10) Note that the list of favorite theoretical virtues includes, foremost, simplicity and fruitfulness (F53's name for unifying power). The "Walrasian" virtues of logical completeness and consistency are ranked lower, while mathematical elegance, another popular theoretical virtue, is not mentioned at all.

F53 thus conforms to the popular solution to the underdetermination conundrum: it cites theoretical virtues as the remedy. Because empirical evidence is insufficient for deciding which theories to accept and reject, we need to supplement empirical virtues with theoretical virtues. When choosing between empirically equivalent theories, we 
choose the one that is theoretically more virtuous. Arbitrariness becomes eliminated or at least reduced.

This invites two observations. First, the argument from theoretical virtues is a standard move amongst realists against those anti-realists who infer from observational equivalence to epistemic equivalence and arbitrariness. F53 would hence seem to stand in the realist camp on this issue, the point being that of all the observationally equivalent theories, we are to believe the one that is simplest and has maximum unifying power. On the other hand, an instrumentalist may argue that such theoretical virtues are epistemically irrelevant and thus fail to be of help in guiding the allocation of belief, in driving theory choice towards the most truthlike theory. It might therefore seem that in matters of underdetermination, F53 holds an indeterminate position between realism and instrumentalism. I can think of one insight in F53 that might be used for making the position more determinate: we have seen that F53 contains the realist idea of ontological unification (in contrast to the instrumentalist idea of mere derivational unification). Since F53 cites unification (or fruitfulness) as a major theoretical virtue, we may conclude that it might be, after all, inclined towards a realist solution to the underdetermination challenge.

The second observation about the position taken by F53 is this: even though the conundrum of underdetermination can be alleviated by means of an appeal to theoretical virtues, theory appraisal will not thereby turn into a fully objective rule-governed affair. As we saw, simplicity and fruitfulness are "notions that defy completely objective specification" and cannot therefore completely eliminate all arbitrariness involved in theory choice. As we will see next, this is a prominent theme in F53 even though it has been overlooked by most readers.

\section{Subjective and social dimensions of theory appraisal}

Those who have read F53 as a positivist or falsificationist statement of economics as a strictly rule-governed and evidence-constrained endeavor will be disappointed upon being pointed out a few passages, completely ignored by most commentators. If one takes the methodology of positive economics to amount to a set of fixed and explicit rules for 
reasoning from given evidence (or evidence fixed by convention or agreement), one should be frustrated by the statements in F53 that emphasize the role of subjective judgment, of the background of economists, of tradition and disciplinary institutions, and of consensus amongst them. These statements in F53 further fortify the concession that strict predictivist tests are unavailable in economics. These statements do not appeal just to theoretical virtues such as simplicity and unifying power but rather to the social nature of scientific work, its embeddedness in academic and other institutions.

Here is a representative passage, making reference to something like the methodological culture of economics in comparison to that of sociology:

Of course, neither the evidence of the economist nor that of the sociologist is conclusive. The decisive test is whether the hypothesis works for the phenomena it purports to explain. But a judgement may be required before any satisfactory test of this kind has been made, and, perhaps, when it cannot be made in the near future, in which case, the judgement will have to be based on the inadequate evidence available. In addition, even when a test can be made, the background of the scientists is not irrelevant to the judgements they reach. There is never certainty in science, and the weight of evidence for or against a hypothesis can never be assessed completely "objectively." The economist will be more tolerant than the sociologist in judging conformity of the implications of the hypothesis with experience, and he will be persuaded to accept the hypothesis tentatively by fewer instances of "conformity". (30; emphases added)

"Complete objectivity" in testing a theory is thus unattainable: judgment and persuasion are involved, and they are directed by the background of the scientist and the degree of tolerance characteristic of the disciplinary culture. In passing, F53 uses falsificationist jargon by referring to the "repeated failure to be contradicted" as evidence for a theory, but it simultaneously acknowledges the role of social factors in shaping the fate of a hypothesis. It recognizes the tenacity with which hypotheses are held against negative evidence and the powerful role of tradition and continued use in creating the image of an acceptable hypothesis: the discipline has its own epistemically forceful folklore that supports a theory but is irreducible to an explicit list of successful empirical tests. Here goes another key passage: 
[The evidence for the maximization-of-returns hypothesis] is extremely hard to document: it is scattered in numerous memorandums, articles, and monographs concerned primarily with specific concrete problems rather than with submitting the hypothesis to test. Yet the continued use and acceptance of the hypothesis over a long period, and the failure of any coherent, self-consistent alternative to be developed and be widely accepted, is strong indirect testimony to its worth. The evidence for a hypothesis always consists of its repeated failure to be contradicted, continues to accumulate so long as the hypothesis is used, and by its very nature is difficult to document at all comprehensively. It tends to become part of the tradition and folklore of a science revealed in the tenacity with which hypotheses are held rather than in any textbook list of instances in which the hypothesis has failed to be contradicted. (22-23; emphases added)

What F53 suggests here is that the social performance (embeddedness in disciplinary institutions and culture, continued use, collective acceptance, tenacious grip) of a theory provides indirect evidence in its support. The core idea is that acceptances and rejections of theories are not strictly rule-governed responses to direct empirical evidence, they rather depend on the subjective judgments of economists whose behavior is shaped by their background and social context. Part of the reason for this can be traced to the character of the rules of application that are described as concrete and incomplete: "The rules of using the model ... cannot possibly be abstract and complete. They must be concrete and in consequence incomplete ..." (25). It is this incompleteness that creates room for judgment and social shaping: no matter how explicitly we succeed in formulating the rules "there inevitably will remain room for judgment in applying the rules. ... It is something that cannot be taught; it can be learned but only by experience and exposure in the 'right' scientific atmosphere, not by rote" (25). The set of rules is embedded in concrete examples and it escapes explicit formulation: it is "mostly implicit and suggested by example" (35-36). ${ }^{8}$

\footnotetext{
${ }^{8}$ These are ideas that would be made more widely known by Thomas Kuhn in his 1962 book, The Structure of Scientific Revolutions. On rules of application, F53 anticipates Kuhn's idea: the rules of application are socially shaped, linguistically inexplicable, and embedded in a previous archetypical concrete examples of scientific practice. The orthodox positivist idea of correspondence rules as explicit components of a theory governing its use is replaced by Kuhn by the notion of a concrete exemplar whose implicit guidance of theory application is brought into scientists' awareness only through the socialization process of scientific education. I first pointed out the Kuhnian features of the account given by F53 in Mäki 1986 (of which an earlier version was published as a discussion paper in 1981).
} 
It is then only natural to wonder whether the acknowledgement of the social shaping of theory appraisal is in tension with the optimism about the prospects of "positive economics" expressed in the opening pages of F53. The suspicion is that once you let the social influences in, the values characteristic of "normative economics" are included in the package and will play a role in undermining hopes of empirically grounded consensus. Whatever consensus there is among economists may emerge as a mixture of empirical, institutional, and ideological influences. The empirical control of belief turns out to be regrettably weak, because of which theories exhibit remarkable capacities of survival.

The denial to economics of the dramatic and direct evidence of the "crucial" experiment does hinder the adequate testing of hypotheses; but this is much less significant than the difficulty it places in the way of achieving a reasonably prompt and wide consensus on the conclusions justified by the available evidence. It renders the weeding-out of unsuccessful hypotheses slow and difficult. They are seldom downed for good and are always cropping up again. (11)

Whatever label one may wish to use for these ideas - pragmatism, collective conventionalism, or social constructivism - it is obvious that they reflect, and resonate with, the practitioner's actual experience that cannot be easily corrupted by positivist or falsificationist textbook teachings about the scientific method. These statements convey highly agreeable insights into the "imperfect" - yet perfectly human - reality of scientific work. But this has a price: tensions emerge with some other ingredients of the image of economics argued by F53. In my rereading, the above valuable observations about the social and institutional nature of economic inquiry will be given precedence.

\section{How to rewrite F53}

I keep admiring F53 for its insightfulness and its capacity to give rise to intriguing questions and to suggest challenging answers. F53 is a rich source of issues and observations that may help us understand important aspects of the intellectual endeavor of economics in its institutional setting. As I have indicated in the footnotes, F53 was ahead of its time in regard to many issues: falsification, comparative testing, unification, 
underdetermination, pragmatics of theorizing, social shaping of scientific reasoning. ${ }^{9}$ At the same time, it appears to share the old methodological wisdom in economics: theories seek to isolate what are believed to be causally significant aspects of social reality.

If we take the F-Mix to be based on ambiguities, inconsistencies and confusions in F53, and if we have a taste for a coherent and defensible account of economic methodology, then the task will be to remove such flaws in F53 and to develop its more promising ingredients into a coherent methodology of economics. This is a project of elimination, reinterpretation, and amendment: one of rereading and rewriting F53. I believe a solid and sensible methodology will emerge as a result of such an exercise. A rewritten F53 will also create new common ground for many participants in the controversies over F53 itself and over economics and its methodology more generally. Within such a shared framework, the remaining disagreements can then be debated in a more focused manner.

I have reread F53 by focusing on a selected set of ambiguities that open up opportunities for re-interpretation. I have exploited these opportunities by highlighting the partly hidden realism in F53's conception of economic science. On this basis, F53 could be rewritten as an unambiguous and consistent realist manifesto. It conveys a methodology of economics that conforms to the tradition of viewing theories or models as partial but potentially true descriptions of causally significant mechanisms. Their primary service is to convey explanatory understanding (answers to why- and howquestions) and only secondarily to yield predictions (answers to what-, when-, and wherequestions). The tradition runs from Senior, Mill and Cairnes to Menger, Marshall and Robbins up to present day economics. In regard to this matter, the often-heard claim about a radical rupture from the Robbinsian to the Friedmanian regime in mainline economic methodology seems exaggerated. What does change is the sophistication with which it is now possible to put some of the key ideas such as the following.

For the purposes of pursuing significant truths by theory, many of its assumptions are allowed to be false. More strongly, false assumptions are required for true theories.

\footnotetext{
${ }^{9}$ Based on this observation, I would take partial departure from the conventional claim that F53 is an example of amateur philosophy of science. My qualified judgment is that F53 contains a set of amazingly sophisticated philosophical insights about scientific theorizing, while at the same time failing to put them together in the form of a coherent system. Rewriting is required precisely in order to remedy this failure.
} 
The best theories are simple - thus violate comprehensive truth - and unify large classes of apparently diverse phenomena. Such phenomena are ontologically unified by explaining them as manifestations of the same simple causal structures. So much for the ontological underpinnings and veristic ambitions of economic theorizing. Another set of sophistications pertains to epistemological concerns, the issues related to assessing theory candidates. One is supposed to test theory candidates by their comparative predictive and explanatory performance, but this will never yield conclusive results. Theory choices are underdetermined by evidence, and they are shaped but not fully determined by theoretical virtues, thus room is created for various subjective judgments as well as social constraints that reflect the institutional structure of economic inquiry. The recognition of this requires a social epistemology involving a strong fallibilism. Of these, fallibilism recommends modesty and awareness of the possibility of being mistaken, while social epistemology recommends critically assessing and redesigning the institutions of economic inquiry so as to improve their capacity in facilitating the acquisition of significant truths about economic reality.

The outcome of this exercise in brief is this: the rewritten F53 = realism + fallibilism + social epistemology. According to this methodology, excellent economists are epistemically ambitious in seeking deep truths about underlying causal structures, and at the same time epistemically modest in acknowledging the possibility of error and the shaping of inquiry by academic and other institutions - and again epistemically ambitious in demanding the design and implementation of institutions that are adequate for enhancing the acquisition of significant truths. This combination is not easy to keep in balance. For perfectly sound reasons, therefore, F53 claims: "More than other scientists, social scientists need to be self-conscious about their methodology." (40)

\section{From reception to the author's intentions?}

Even though the foregoing has been an exercise in reception methodology, a final speculation may be permitted about how all this might relate to the intentions and beliefs of the author of F53. I have argued that F53 fails to unambiguously express any coherent set of beliefs and intentions about economic methodology. The unavailability of a single 
coherent methodology in F53 is consistent with two possibilities concerning Milton Friedman's beliefs and intentions. The first possibility is that Friedman did not have a coherent methodology in mind and that F53 reflects this fact accurately (for a rhetorical account of this option, see Mäki 1986). The second possibility is that Friedman had a coherent methodology in mind but just failed to convey it in F53 due to flaws in textual production.

Whatever the case, the ambiguities and inconsistencies in F53 have enabled multiple receptions and mutually incompatible interpretations in the long series of commentaries. In a special sense, we might say that any such interpretation is underdetermined by textual evidence: the textual evidence does not fix any of the interpretations as the correct one. However, this is not a situation of underdetermination in precisely the same sense that was recognized by F53 itself as discussed above: namely that an infinite number of theories are consistent with the same set of data. Here, various interpretations of F53 rely on reading selected passages rather than the textual data in its entirety: the selected sets of evidence overlap but are not identical (for example, most of the instrumentalist interpretations of F53 have neglected those passages that are crucial for my realist and social constructivist readings). This is bound to be so as no interpretation is possibly consistent with the textual data as a whole. What I am envisaging here is not exactly the same idea as the standard argument from underdetermination -- but it may have interesting affinities with actual situations in economics and elsewhere in science!

In a sense, then, the economic methodology possibly intended by the author of F53 is underdetermined by F53 itself and is therefore open for multiple interpretations. There is just one way I can think of that might help reduce this multiplicity: analyze Friedman's other statements on methodology, and analyze Friedman's work in economics for its implicit methodology. This is a task for historians of economic methodology interested in one Nobel Laureate's views - not a task for reception methodology in the sense pursued here. Yet, were these two perspectives combined, I would not be surprised if it turned out to be the case that F53 as rewritten along the lines suggested above is the methodological statement that the author of F53 intended to write. 


\section{References}

Boland, Lawrence (1979). A critique of Friedman's critics, Journal of Economic Literature 17, 503-522

Caldwell, Bruce (1992). Friedman's methodological instrumentalism: A Modification, Research in the History of Economic Thought and Methodology 10, 119-128

Duhem, Pierre (1954). The Structure of Physical Theory. Princeton: Princeton University Press

Friedman, Michael (1974). Explanation and scientific understanding, Journal of Philosophy, 71, 5-19

Friedman, Milton (1953). The methodology of positive economics, pp. 1-43 of his Essays in Positive Economics. Chicago: Chicago University Press

Hands, D. Wade (2003). Did Milton Friedman's methodology license the Formalist Revolution?, Journal of Economic Methodology, 10, 507-520

Hindriks, Frank (2005). Unobservability, tractability, and the battle of assumptions, Journal of Economic Methodology, 12, 383-406

Hirsch, Abraham and DeMarchi, Neil (1990). Milton Friedman. Economics in Theory and Practice. Hertfordshire: Harvester Wheatsheaf

Kitcher, Philip (1981). Explanatory unification, Philosophy of Science, 48, 507-531

Kuhn, Thomas S. (1970/1962). The Structure of Scientific Revolutions. Chicago: University of Chicago Press

Mäki, Uskali (1986). Rhetoric at the expense of coherence: A reinterpretation of Milton Friedman's methodology, Research in the History of Economic Thought and Methodology 4, 127-143

(1992). Friedman and realism, Research in the History of Economic Thought and Methodology 10, 171-195

(1998). "As if", in The Handbook of Economic Methodology, ed. J. Davis, W. Hands and U. Mäki. Edward Elgar 1998, 25-27

(2000) Kinds of assumptions and their truth: Shaking an untwisted F-twist, Kyklos, $53,303-322$

(2001). Explanatory unification: Double and doubtful, Philosophy of the Social Sciences, 31, 488-506

(2003). 'The Methodology of Positive Economics' (1953) does not give us the methodology of positive economics, Journal of Economic Methodology, 10, 495505

(2004). Realism and the nature of theory: A lesson from J.H. von Thünen for economists and geographers, Environment and Planning A, 36, 1719-1736

Mayer, Thomas (1993). Friedman's methodology of positive economics: A soft reading, Economic Inquiry 31, 213-223

Musgrave, Alan (1981). 'Unreal assumptions' in economic theory: The F-twist untwisted, Kyklos 34, 377-387 
Rotwein, Eugene (1959). On the methodology of positive economics, Quarterly Journal of Economics, 73, 554-575

Samuelson, Paul (1963). Problems of methodology - Discussion, American Economic Review, Papers and Proceedings, 53, 231-236

Wong, Stanley (1973). The F-Twist and the methodology of Paul Samuelson, American Economic Review, 63, 312-325 